\title{
La rentabilité de la production de pomme de terre dans la région des sols de laves au Rwanda
}

\author{
Aristide Maniriho, Fabio Berti, Edouard Musabanganji \& Philippe Lebailly
}

Aristide Maniriho : Rwandais, Diplôme de Master of Science in Economics, Assistant Lecturer à l'Ecole d'Economie, Uinversité du Rwanda; Doctorant à l'Unité d'Economie et de Développement Rural, Gembloux Agro-Bio Tech, Université de Liège, Passage des Déportés, 2 B-5030 Gembloux, Belgique. Auteur correspondant Email: manirihoaristide1@gmail.com

Fabio Berti : Belge, Diplôme d'ingénieur agronome, Chargé de Recherche et Projets à l’Unité d’Economie et de Développement Rural, Gembloux Agro-Bio Tech, Université de Liège, Passage des Déportés, 2 B-5030 Gembloux, Belgique. Email: fabio.berti@uliege.be

Edouard Musabanganji : Rwandais, Docteur en Sciences Agronomiques et Ingénierie Biologique, Senior Lecturer à l’Ecole d'Economie, Université du Rwanda, KK 737 Street, Kigali, Rwanda. Email: musabanganji@gmail.com

Philippe Lebailly : Belge, Docteur en Sciences Agronomiques et Ingénierie Biologique, Professeur à l'Unité d’Economie et de Développement Rural, Gembloux Agro-Bio Tech, Université de Liège, Passage des Déportés, 2 B-5030 Gembloux, Belgique. Email: philippe.lebailly@ulige.be

DOI: $10.25518 / 2295-8010.1909$

\section{Résumé :}

Cet article vise à analyser la rentabilité des petits exploitants de la pomme de terre dans la région des sols de laves au Rwanda. Les données utilisées ont été collectées auprès de 132 producteurs choisis de façon aléatoire. Les méthodes d'analyse budgétaire, de la comparaison de la valeur de la production aux couts de production, et d'analyse corrélationnelle ont été employées dans l'analyse des données. Les résultats de l'analyse budgétaire montrent que la production de la pomme de terre est rentable et que la rentabilité est sensible à une baisse de rendement ainsi qu'à une hausse des coûts de production. Par comparaison, l'analyse de sensibilité montre que les effets de la diminution de l'offre sur le revenu net sont plus néfastes que ceux de l'augmentation du coût de production. Pour améliorer le niveau de rentabilité, les producteurs de pomme de terre devraient adopter des pratiques agricoles susceptibles de booster la productivité. L'analyse corrélationnelle met en évidence une relation positive et significative entre le revenu agricole net et les autres indicateurs, notamment l'expérience de l'exploitant et la surface des terres exploitées. Ces variables sont des facteurs qu'il faut considérer pour maintenir et/ou améliorer le niveau de rentabilité agricole.

Mots-clés : rentabilité, pomme de terre, revenu net agricole, région des sols de laves, Rwanda

\section{Abstract :}

The profitability of potato production in the volcanic highlands in Rwanda

This article aims to analyze the profitability of potato small-scale producers in Volcanic 
La rentabilité de la production de pomme de terre dans la région des sols de ...

Highlands in Rwanda. Data used were collected from a random sample of 132 producers. Different methods namely accounting method, cost-benefit analysis and correlation analysis were used in the analysis of data. The results from the budgetary analysis show that the potato production is profitable and that the profitability is sensitive to the shock of production and the increase in the cost of production. By comparison, the results from sensitivity analysis of net farm income show that a decrease in potato production generates more effects than the increase in the cost of fertilizers. To improve the level of profitability, potato growers must adopt farming practices that boost the productivity. By the correlational method, the coefficient is positive and significant between net farm income and other factors namely the cost of production, the selling price, the farmer's experience and the size of the cultivated land. These are factors that must be considered to maintain and/or improve the level of agricultural profitability.

Keywords : profitability, potato, net farm income, volcanic highlands, Rwanda

\section{Introduction}

La pomme de terre (Solanum tuberosum) constitue un aliment de base pour les deux tiers de la population mondiale et occupe la troisième place parmi les légumes les plus consommés dans le monde, étant donné que ce tubercule produit plus d'énergie que tout autre type de légume pour la nutrition humaine (3). Hautement recommandée par l'Organisation des Nations Unies pour l'Agriculture et l'Alimentation (FAO) comme culture de sécurité alimentaire (37), la pomme de terre se place parmi les premières cultures vivrières en termes de composante de la ration alimentaire de base (11) et occupe la quatrième place après le maïs, le riz et le blé (44).

La pomme de terre aide les petits fermiers à surmonter les problèmes de faim et de nutrition car il s'agit d'une culture à haut rendement et à courte saison de croissance dont le cycle de culture peut varier de 100 à 120 jours, selon les variétés et les conditions agro-écologiques. En Afrique, les grands pays producteurs de pomme de terre en volume produit sont l'Egypte, le Malawi, l'Afrique du Sud, l'Algérie, le Maroc, le Rwanda (sixième place), le Nigeria, le Kenya, l'Ouganda, l'Angola et l'Ethiopie (13), le Rwanda étant donc le premier producteur en Afrique de l'Est, avant le Kenya et l'Ouganda.

La pomme de terre a été introduite au Rwanda par des missionnaires allemands à la fin du 19ème siècle (42). Avec d'autres plantes, notamment la patate douce et le manioc, ces trois principales racines et tubercules contribuaient à $56 \%$ de la production agricole totale au Rwanda en 2001 (29). Les cultures vivrières dominent l'agriculture rwandaise et la pomme de terre est très importante pour la génération de revenus et la sécurité alimentaire nationale (5). La consommation annuelle de pommes de terre est très élevée : $145 \mathrm{~kg}$ par personne et par an. Il s'agit de la deuxième source d'énergie alimentaire pour les ménages après le manioc (39). Bien que la pomme de terre soit très importante au Rwanda, les rendements moyens restent faibles, variant entre 5 et 20 tonnes / ha par rapport à des rendements potentiels de 30 tonnes / ha $(15,30)$. Les variétés les plus exploitées sont entre autres Cruza, Gikungu, Kigega, Kinigi, Kirundo, Mabondo, Mizero et Sangema. La production de la pomme de terre est favorable dans les sols fertiles, légers, profonds, bien drainés et légèrement acides ( $\mathrm{pH} 6$ à 6,5), entre 1800 à 2300m d'altitude, les températures optimales variant entre 12 et $20^{\circ} \mathrm{C}$, avec des pluies régulières de $\pm 300 \mathrm{~mm}$ tout au long du cycle de production (1). Parmi les causes des rendements sous-optimaux en milieu paysan, figurent les maladies du mildiou, du flétrissement bactérien, ainsi que de la dégénérescence des plants de pomme de terre, causées par des virus et des mycoplasmes (15). Les statistiques récentes au Rwanda (35) montrent que la 
production de la pomme de terre s'élève à 396.064 tonnes pour la saison 2018 B1 , dont plus de 80 $\%$ est issue des provinces du Nord et de l'Ouest, alors que les autres $20 \%$ sont issus d'exploitations marginales très dispersées dans les provinces du Sud, de l'Ouest et la Ville de Kigali. Il faut signaler aussi qu'environ $10 \%$ de la production est consommée par les producteurs (voir Figure 1).

\section{Utilisation de la production de la pomme de terre}

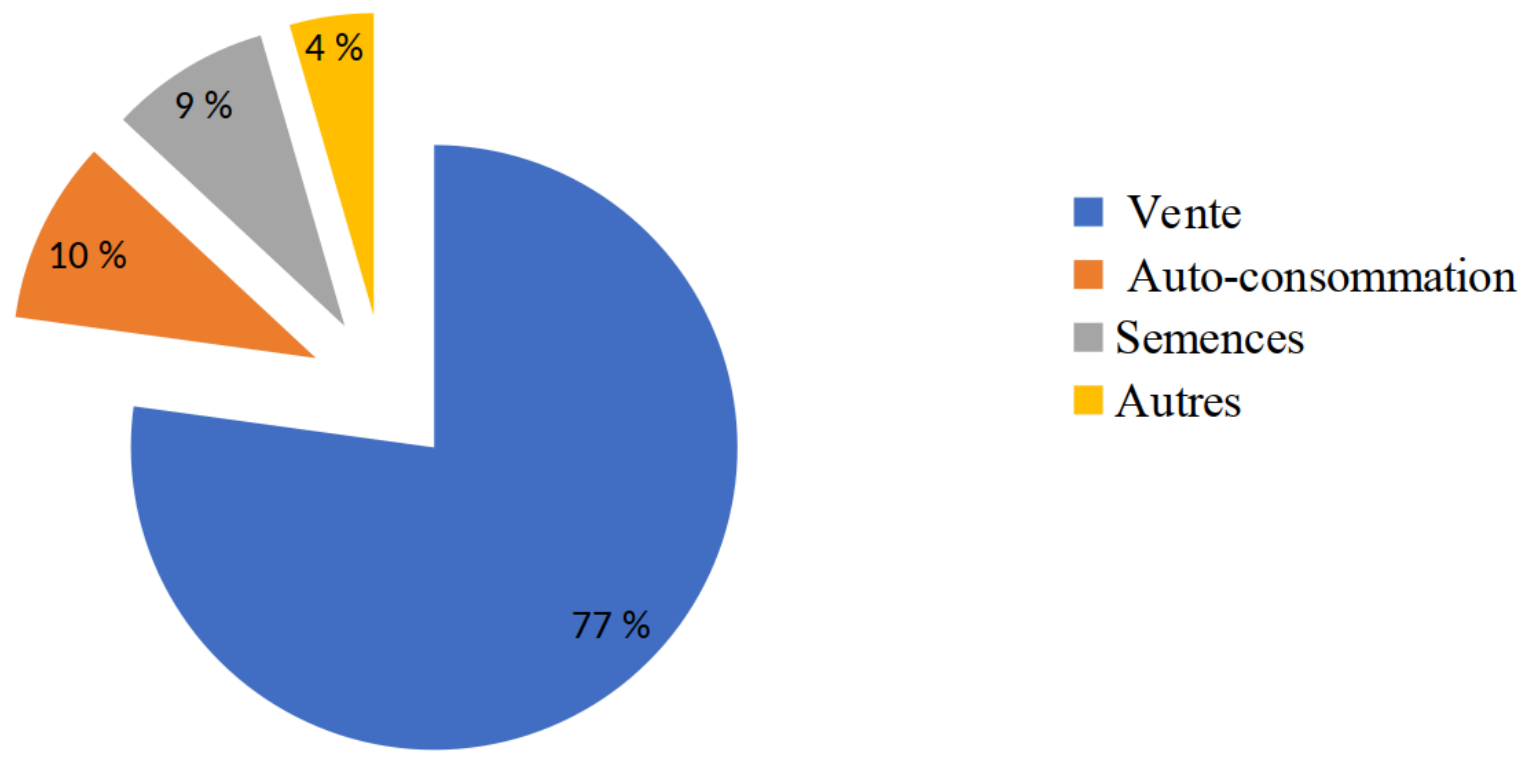

Figure 1. Différentes affectations de la production de pomme de terre dans la région des sols de laves au Rwanda.

Source : Notre enquête octobre - décembre 2019

En vue de permettre le développement des exploitations agricoles, la rentabilité de l'activité agricole est essentielle. De même, Corselius et al. (9) ont justifié la nécessité d'une rentabilité agricole et ont souligné que la rentabilité permet aux agriculteurs de répondre à des niveaux croissants de demande et de soutenir un niveau de vie acceptable tout en garantissant les investissements annuels nécessaires pour améliorer progressivement la productivité des ressources.

C'est ainsi que, dans son manifeste de développement agricole intitulé «Crop Intensification Program » ou CIP adopté en septembre 2009, le gouvernement rwandais a inclus la pomme de terre, le maïs, le riz, le manioc, le haricot et le blé parmi les cultures prioritaires $(2,28)$. Peu après, il a été décidé de promouvoir surtout la pomme de terre pour en tirer des devises en renforçant ses exportations (36). Malgré cela, le Rwanda reste jusqu'à présent importateur net de quelques produits agricoles, tels que la pomme de terre, le maïs-grain, le riz et le soya (32).

Un peu avant l'adoption du programme d'intensification agricole (CIP), Mugabe et al. (30) ont analysé le niveau de rentabilité des différentes cultures dans toutes les zones agro-écologiques du Rwanda. Maniriho \& Bizoza (23) ont examiné la rentabilité des petites exploitations agricoles dans le District de Musanze en procédant par la méthode budgétaire. Les résultats de l'analyse de sensibilité révèlent que la rentabilité de la pomme de terre est sensible à l'augmentation des 
La rentabilité de la production de pomme de terre dans la région des sols de ...

dépenses opérationnelles, à l'augmentation du prix de vente, ainsi qu'à la baisse de la production. Par l'analyse de la compétitivité du secteur agricole, Musabanganji et al. (32) ont établi que le Rwanda reste un importateur net de produits agricoles prioritaires, la pomme de terre incluse. Toutes ces informations seraient aussi un révélateur de problèmes courants de productivité et d'efficience et, par conséquent, d'un déficit de rentabilité dans le secteur agricole rwandais.

En référence aux efforts significatifs de développement entrepris dans le secteur agricole au Rwanda, il s'avère nécessaire de montrer si ces résultats restent toujours valables. De plus, la majorité des études réalisées ont pris un seul district comme cas d'étude. Il est alors important de comparer la rentabilité entre les différents districts et entre les différentes catégories d'exploitants agricoles dans une zone agro-écologique donnée, telle que celle de «Birunga » (région agricole des sols de laves) que nous avons retenue. Il s'agit donc d'une analyse qui s'intéresse aux effets de l'activité agricole sur le revenu des agriculteurs et analyse la variabilité temporelle en nous référant à une étude de 2009 (26), ce qui nous permettra d'évaluer la contribution de la politique agricole sur le revenu des producteurs de la pomme de terre. D'autres politiques agricoles et sociales considérées sont, entre autres, le Programme Vision 2020 Umurenge (VUP, acronyme anglais) adopté en 2007 (18), le Programme Umurenge pour l'Epargne et le Crédit (USACCO, acronyme anglais), initié en 2009 (38), et le Programme « une vache par famille pauvre » aussi connu comme «Girinka », adopté en 2007 (20). Par ailleurs, notre analyse se réfère à l'étude de Mugabe et al. (30) sur la rentabilité des différentes cultures dans toutes les zones au Rwanda, considérée comme «baseline ». Nous avons actualisé (10 ans plus tard) les données de cette étude pour montrer si les résultats obtenus sont toujours valables. Comme le Ministère de l'Agriculture et de l'élevage au Rwanda (MINAGRI) l'explique (27), pour beaucoup d'agriculteurs actuellement au Rwanda, les exploitations sont trop petites pour produire un excédent commercialisable et, par conséquent, elles ne peuvent pas sortir de la pauvreté ou de la malnutrition. Certains peuvent trouver un emploi hors ferme, mais ils auront souvent besoin d'un soutien par le biais de programmes de protection sociale.

La présente recherche s'efforce donc de souligner le rôle des petites exploitations de pomme terre dans le développement agricole au Rwanda. En tant que culture à production spéculative, la question revient alors de savoir comment la petite exploitation de pomme de terre peut contribuer à la consolidation économique des producteurs. Se fondant sur les différents enjeux liés aux exploitations agricoles, cette étude a analysé la performance économique (rentabilité) des petites exploitations de pomme de terre dans la région des sols de laves au Rwanda, dans le cadre de la spécialisation régionale de l'agriculture.

La rentabilité (ou encore la profitabilité) est couramment définie comme l'aptitude générale de l'exploitation à dégager une marge bénéficiaire ; elle donne un niveau potentiel de performance qui est souvent posé comme le différentiel entre prix de vente et prix de revient. Il s'agit ici de la capacité de l'exploitation à générer un bénéfice à partir des capitaux investis (4). Du fait que les indicateurs de rentabilité (tels que la marge brute (MB) et la valeur ajoutée (VA)) ne garantissent pas que les producteurs couvrent la totalité des dépenses et dégagent ainsi un profit, nous avons aussi considéré les indicateurs tels que le revenu net $(\mathrm{RN})$ pour déterminer le niveau de profit de l'activité agricole $(9,16)$. Tous ces indicateurs ont été estimés pour les trois catégories de producteurs, notamment les producteurs les moins performants, les producteurs moyennement performants, et les producteurs les plus performants.

Pour atteindre l'objectif de notre étude, nous avons procédé à la détermination du revenu total, des consommations intermédiaires, du coût variable total, du coût fixe total, des coûts totaux, de la 
marge brute, de la valeur ajoutée, et du revenu net.

\section{Matériels et méthodes}

\subsection{Collecte des données}

Les données de cette étude ont été collectées à l'aide d'un questionnaire d'enquête administré à un échantillon de 132 producteurs de pomme de terre dans la Région Agricole des Sols de Laves au Rwanda, d'octobre à décembre 2019. Pour avoir un échantillon représentatif, nous avons procédé à l'échantillonnage aléatoire simple recommandé par Magain (22). Notre échantillon a été formé en trois étapes. Lors de la première étape, nous avons sélectionné de façon aléatoire un secteur administratif dans chacun des quatre Districts couvrant la région d'étude. Les secteurs de Gahunga, Kinigi, Bigogwe et Nyakiriba sont choisis respectivement dans les districts de Burera, Musanze, Nyabihu et Rubavu. Lors de la deuxième étape, deux ou trois cellules 2 sont désignées au niveau de chaque secteur. Le choix des cellules a été raisonné par deux principaux critères, notamment $\left(1^{\circ}\right)$ la pomme de terre y est exploitée en tant que culture prioritaire dans le cadre du programme national d'intensification agricole et $\left(2^{\circ}\right)$ la cellule est facilement accessible (c'est-à-dire connectée au réseau national routier). Pour la troisième étape, nous avons administré notre questionnaire aux producteurs de pomme de terre dans les cellules choisies, ce qui nous a conduits à un échantillon de 132 producteurs à la fin de l'enquête.

Nous avons collecté les données sur la parcelle principale de chaque producteur où une culture pure s'applique au cours de la saison culturale 2019 B. La sélection des parcelles principales a porté sur l'un ou l'autre des trois critères: $\left(1^{\circ}\right)$ soit l'exploitation est principale en termes de taille, $\left(2^{\circ}\right)$ soit principale en terme de production, $\left(3^{\circ}\right)$ soit l'exploitation répond à ces deux critères simultanément.

Les éléments du questionnaire portaient sur les caractéristiques socioéconomiques des producteurs et de leurs ménages, la taille de l'exploitation, les informations en rapport avec la main d'œuvre, les intrants, les pesticides, l'équipement et outillage agricole, la rente (coût d'accès à la terre), le coût du transport, ainsi que la production et le prix de vente.

\subsection{Présentation du milieu d'étude}

Notre étude couvre la Région agro-écologique des Sols de Laves, l'une des 12 zones agroécologiques au Rwanda (40). Cette région agro-écologique, encore appelée Birunga, est bien connue pour son sol essentiellement à vocation agricole (altitude de 1600 à $2500 \mathrm{~m}$, avec des sols volcaniques de couleur noire, très perméables, dont la valeur agricole est excellente) (34). Les spéculations principales qui y sont rencontrées sont la pomme de terre, les légumes (oignon rouge, oignon blanc, etc.), le maïs, le haricot, le blé, ... (27). La figure 2 localise la zone d'étude, « Région agricole des sols de laves » au Rwanda qui s'étend dans les Districts de Burera, Musanze, Nyabihu et Rubavu. 


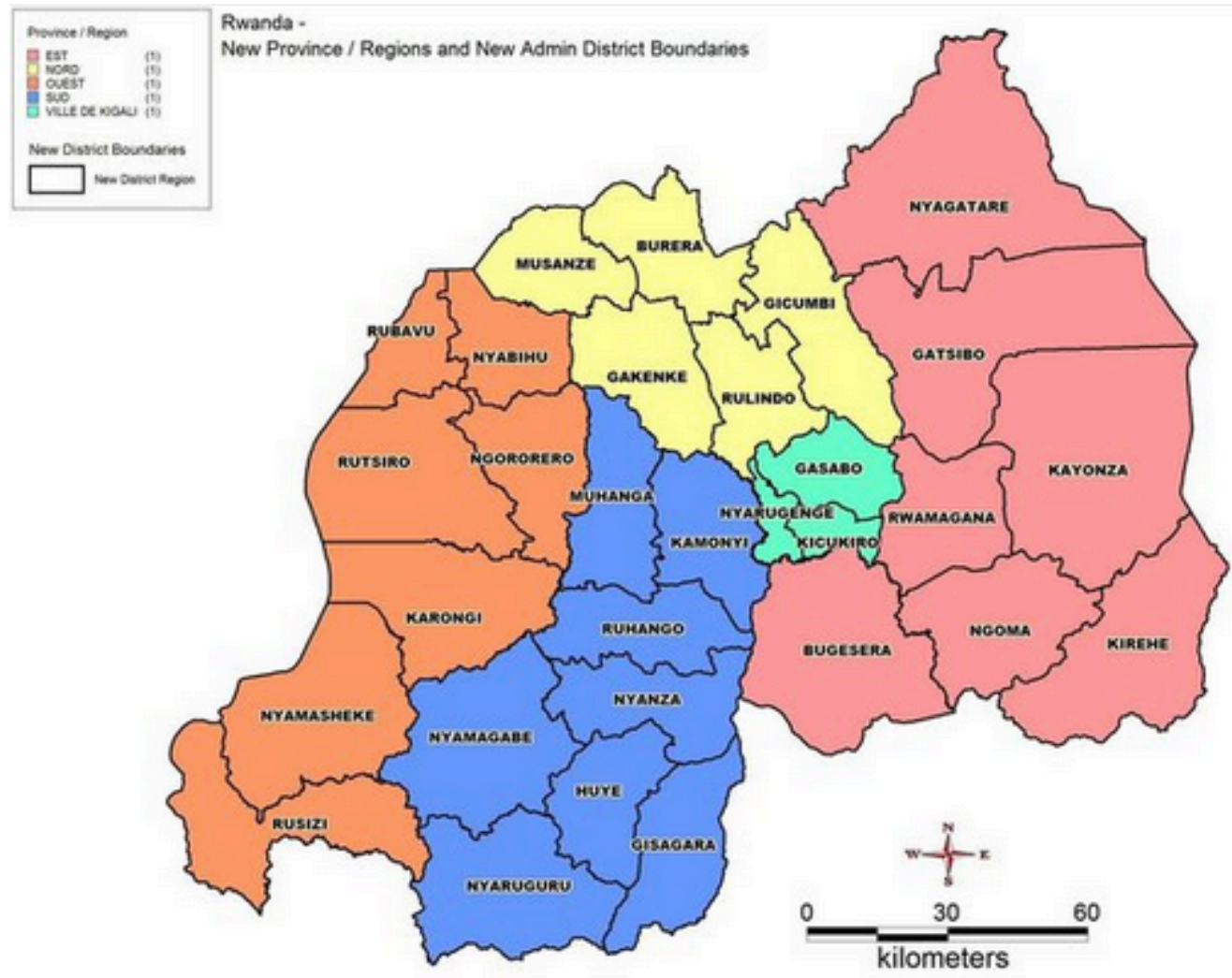

Figure 2. Localisation de la Région agricole des sols de laves sur la carte du Rwanda (Districts de Rubavu, Nyabihu, Musanze et Burera)

\subsection{Méthodes d'analyse des données}

Dans l'analyse des données, nous avons utilisé la méthode budgétaire (calcul du Produit Brut, de la Marge Brute («Gross Margin »), et du Revenu Agricole Net, (« Net Farm Income ») (16). Le produit brut ou Revenu Total (RT), la marge brute (MB), le revenu net (RN) et le coût total sont respectivement calculés à l'aide des formules de (i) à (v). Les indicateurs de productivité et de rentabilité ont été ramenés par hectare pour permettre la comparaison entre les catégories des producteurs.

$\mathrm{RT}=\Sigma\left(P_{i}^{*} Q_{i}\right)(\mathrm{i})$

$\mathrm{MB}=\Sigma\left(P_{i}^{*} Q_{i}\right)-\Sigma X_{i} P_{x i}=\mathrm{RT}-\mathrm{CTV}(\mathrm{ii})$

$\mathrm{RN}=\Pi=\mathrm{RT}-\mathrm{CT}=\Sigma\left(P_{i}^{*} Q_{i}\right)-(\mathrm{CTV}+\mathrm{CTF})(\mathrm{iii})$

$\mathrm{CT}=\mathrm{CTF}+\mathrm{CTV}(\mathrm{iv})$

Où $\mathrm{RT}=$ revenu total (produit brut), $P_{\mathrm{i}}=$ prix de vente du produit agricole $i$, et $\mathrm{Q}_{\mathrm{i}}=$ quantité totale produite du produit agricole $i, \mathrm{CT}=$ coût total, CTV=coût variable total, $\mathrm{CTF}=$ coût fixe total, $X$ est la matrice des inputs, $P_{\mathrm{X}}$ est la matrice des prix des inputs, $\Sigma$ est un signe de sommation, RN est le revenu net (encore appelé profit et noté, п). Les indicateurs décrits par les équations (i) à (iv) 
sont complétés par la valeur ajoutée (VA) qui est la différence entre la valeur de la production totale (RT) et les consommations intermédiaires (CI) et sert à rémunérer les différents acteurs qui interviennent dans le processus de production : l'État, les institutions de micro crédit, la main d'œuvre, etc. (9). La VA est ainsi définie par l'équation (v).

$\mathrm{VA}=\mathrm{RT}-\mathrm{CI}(\mathrm{v})$

Où les consommations intermédiaires (CI) comprennent les dépenses sur les plants de pomme de terre, les dépenses sur les engrais organiques et chimiques, les dépenses sur les pesticides ainsi que le coût de transport.

Le revenu total (RT) (encore appelé produit brut) est la valeur monétaire de la production totale par unité de surface, généralement par hectare. Pour un producteur produisant un seul produit, cette valeur est obtenue par la multiplication de la quantité produite par le prix de vente unitaire ; pour tous les producteurs, le RT est obtenu en sommant la quantité produite par son prix de vente unitaire quelle que soit l'affectation de la production. Nous n'avons considéré que la valeur du produit primaire de chaque exploitant agricole étant donné que l'estimation de la valeur exacte des produits secondaires n'est pratiquement pas facile à estimer (5) et que leur valeur monétaire est négligeable. Mais nous n'avons pas pu analyser la rentabilité sur l'ensemble des productions associées par manque de données portant sur les saisons précédentes pour les producteurs pris dans notre échantillon.

Le coût total de production (CT) est la valeur de toutes les ressources et tous les services utilisés dans le processus de production. Ceci inclut le coût total variable (CTV) et le coût total fixe (CTF). Pour le cas de notre étude, le coût total fixe est composé des dépenses sur la location de la terre (la rente) et de l'amortissement (dépréciation) du matériel et de l'équipement. La rente de la terre en propriété a été estimé par son coût d'opportunité référence faite à rente payée par les exploitants locataires. Nous n'avons pas inclus les taxes et les intérêts payés dans le coût total fixe : l'État ne prélève pas les taxes au niveau de la production agricole, alors que l'information sur les coûts liés au crédit n'est pas disponible pour tous les producteurs échantillonnés. Pour l'estimation de l'amortissement, nous avons opté pour la proposition de Maniriho (24) d'affecter la durée de vie moyenne des outils et matériels agricoles de 3 ans pour les agriculteurs rwandais de la région d'étude. Le coût total variable inclut les dépenses pour la main-d'œuvre, les engrais chimiques, les engrais organiques, les jeunes plants de pomme de terre, les pesticides et le coût de transport. Étant donné que le coût total croît avec l'augmentation de la production, c'est donc la liaison entre la quantité produite et son coût total de production qui est appelée «fonction de coût » (25).

Le revenu net (ou tout simplement le profit) est la différence entre le revenu total et le coût total (ou dépenses totales) (voir équation iii). Les équations ii et iii respectivement sur la marge brute (MB) et sur le revenu net (RN) signifient aussi que le RN=MB - CTF, où RN est le revenu net qui est un profit (si positif) ou une perte (si négatif).

En complément de ces méthodes, la corrélation entre certaines variables a été calculée. Il s'agit notamment de la corrélation entre le coût de production et le revenu net, et la corrélation entre la surface des terres exploitées et le revenu net. Les résultats de l'analyse corrélationnelle sont présentés dans le Tableau 2.

En termes du niveau de RN, nous avons enfin procédé par l'analyse en grappes ( « cluster analysis ») où la méthode de variance minimale, encore appelée méthode de Ward (47) a été utilisée pour 
La rentabilité de la production de pomme de terre dans la région des sols de ...

former les groupes hiérarchiques des producteurs de pomme de terre mutuellement exclusifs, dont les membres de chacun sont au maximum similaires. Cette méthode est souvent appelée « analyse de groupes » en économie agricole. Les trois catégories ainsi formées sont notamment $\left(1^{\circ}\right)$ les producteurs non performants, $\left(2^{\circ}\right)$ les producteurs moyennement performants, et $\left(3^{\circ}\right)$ les producteurs les plus performants. Ce regroupement a été motivé par le fait que les niveaux de RN des producteurs de pomme de terre sont différents : certains font une perte $(\mathrm{RN}<0)$, les autres couvrent à peine la totalité de leurs dépenses $(\mathrm{RN}=0)$, alors d'autres gagnent un $\mathrm{RN}$ positif $(\mathrm{RN}>0)$. Par conséquent, les interventions publiques nécessaires pour l'amélioration des facteurs explicatifs du revenu net ne seraient pas les mêmes selon qu'on veut apporter des solutions aux problèmes des producteurs moins performants, des producteurs moyennement performants ou à des producteurs plus performants.

\section{Résultats}

Cette section présente les résultats de l'étude en rapport avec les différentes affectations de la production de la pomme de terre, les caractéristiques socioéconomiques des producteurs, la corrélation entre le revenu net des producteurs de la pomme de terre et les variables qui le déterminent, le niveau de rentabilité de la pomme de terre et sa sensibilité à la diminution de l'offre et au changement du coût de production.

Les résultats sur l'utilisation de la production de la pomme de terre (Figure 1) montrent qu'elle est en grande partie (77 \%) vendue, $10 \%$ de la production de la pomme de terre est consommée par les producteurs (autoconsommation), $9 \%$ utilisée comme jeunes plants de pomme de terre pour la prochaine saison, et $4 \%$ sont affectés à différentes fins (dons et aides aux amis et membres de la famille, par exemple).

Les résultats sur les caractéristiques socioéconomiques des enquêtés sont résumés dans le tableau 1. Les statistiques descriptives montrent que la superficie de terre allouée à l'exploitation de la pomme de terre est en moyenne de 0,36 hectare pour la saison 2019 B. La distribution par sexe des producteurs de la pomme de terre dans la zone d'étude est de 70 hommes (53\%) contre 62 femmes (47\%). On remarque aussi que $67 \%$ des producteurs de la pomme de terre pratiquent la rotation des cultures, alors que $33 \%$ ne la pratiquent pas. Dans ce cadre, un producteur à Kinigi, District de Musanze, confirme que l'on peut produire la pomme de terre au moins sur deux saisons culturales consécutives pour maximiser son chiffre d'affaires sur la production, surtout quand la première saison n'a pas été bien rémunératrice, ce qui reflète que la rotation est motivée beaucoup plus par la spéculation que par la logique sur l'ordre des cultures.

Lâge moyen des exploitants agricoles est de 40 ans, ce qui montre que les exploitants sont encore physiquement forts et possèdent en principe l'expérience requise pour bien mener le travail agricole. Le ratio de la production par unité de surface exploitée, soit le rendement exprimé en Kg/ ha a été estimé à $8.173 \mathrm{Kg} / \mathrm{ha}$. En plus, la superficie des terres cultivées est très petite et est égale en moyenne à $3.599 \mathrm{~m}^{2}$ (équivalent à 0,36 hectares), ce qui pourrait affecter significativement le niveau de revenu. Il est également à remarquer que les producteurs de la pomme de terre ont fréquenté l'école et proviennent de tous les niveaux d'étude. Tout autre détail peut se lire dans le tableau 1. 
Tropicultura Tropicultura 2295-8010 Volume 39 (2021) Numéro 4, 1909

Tableau 1. Caractéristiques socioéconomiques des producteurs de pomme de terre dans la zone d'étude. 
La rentabilité de la production de pomme de terre dans la région des sols de ...

\begin{tabular}{|c|c|c|c|}
\hline Variables qualitatives & Effectif & & Pourcentage \\
\hline \multicolumn{4}{|l|}{ Sexe } \\
\hline Masculin & 70 & & 53,03 \\
\hline Féminin & 62 & & 46,97 \\
\hline \multicolumn{4}{|l|}{ Niveau d'éducation } \\
\hline Pas été à l'école & 20 & & 15,27 \\
\hline Primaire non complet & 30 & & 22,9 \\
\hline Primaire complet & 32 & & 24,43 \\
\hline Secondaire non complet & 10 & & 7,63 \\
\hline Secondaire complet & 19 & & 14,5 \\
\hline Technique et vocationnel & 17 & & 12,98 \\
\hline Tertiaire non complet & 1 & & 0,76 \\
\hline Tertiaire complet & 2 & & 1,53 \\
\hline \multicolumn{4}{|l|}{ District } \\
\hline Burera & 33 & & 25 \\
\hline Musanze & 82 & & 62,12 \\
\hline Nyabihu & 11 & & 8,33 \\
\hline Rubavu & 6 & & 4,55 \\
\hline \multicolumn{4}{|l|}{ Rotation des cultures } \\
\hline Bien pratiquée & 63 & & 47,73 \\
\hline Très rarement pratiquée & 25 & & 18,94 \\
\hline Pas du tout pratiquée & 44 & & 33,33 \\
\hline Variables quantitatives & Moyenne & Minimum & Maximum \\
\hline Age & 40 & 21 & 62 \\
\hline Surface cultivée $\left(\mathrm{m}^{2}\right)$ & 3.599 & 800 & 12.000 \\
\hline Production (Kg) & 3.048 & 200 & 15.000 \\
\hline
\end{tabular}




$\begin{array}{llcr}\text { Rendement (Kg/ha) } & 8.173 & 1.333 & 20.000 \\ \text { RT /ha (FRW) } & 1.639 .385 & 200.000 & 4.114 .286 \\ \text { CT/ha (FRW) } & 1.063 .504 & 507.264 & 2.332 .229 \\ \text { RN/ha (FRW) } & 575.881 & -819.500 & 3.256 .619\end{array}$

Note $: \mathrm{m}^{2}=$ mètre carré, $\mathrm{Kg}=$ kilogrammes, $\mathrm{RT}=$ revenu total, $\mathrm{CT}=$ coût de production, $\mathrm{RN}=$ revenu net, ha=hectare, et FRW = francs rwandais. Pour les variables quantitatives, les chiffres sont arrondis à l'unité.

Les résultats de l'analyse corrélationnelle (tableau 2) montrent que le revenu net est positivement affecté par la surface exploitée (coefficient de corrélation, , probabilité, ), que le RN est positivement et significativement corrélé avec la MB (, ), et que la MB est positivement et significativement corrélée avec la surface cultivée (, ). De même, la corrélation entre le revenu net et le coût de production est positive (, probabilité, ), ainsi que la corrélation entre la surface de la terre exploitée et le coût de production (, probabilité, ). Le revenu net est aussi positivement influencé par le prix de vente, ainsi que par le niveau d'expérience des agriculteurs. Une corrélation positive et significative est aussi observée entre la vulgarisation (nombre de visites) et le CT (,), ce qui serait expliqué par l'adoption des variétés à haut rendement et l'usage des intrants modernes. Les résultats montrent aussi que l'expérience est positivement corrélée au $\mathrm{RN}($ (), à la $\mathrm{MB}($,) et au CT (,). De plus, la surface cultivée est positivement corrélée au RN (,), à la MB (,), et au CT (,). Le tableau 2 montre la matrice des coefficients de corrélation entre le revenu net et les variables qui l'influencent. 
La rentabilité de la production de pomme de terre dans la région des sols de ...

Tableau 2. Matrice des coefficients de corrélation entre le revenu net et ses déterminants $(\mathbf{n}=132)$.

\begin{tabular}{|c|c|c|c|c|c|c|c|}
\hline Variables & (1) & $(2)$ & (3) & $(4)$ & (5) & (6) & (7) \\
\hline (1) Revenu net (RN) & 1 & & & & & & \\
\hline \multirow[t]{2}{*}{ (2) Marge brute (MB) } & 0,99 & 1 & & & & & \\
\hline & 0 & & & & & & \\
\hline \multirow[t]{2}{*}{ (3) Coût de production (CT) } & 0,54 & 0,56 & 1 & & & & \\
\hline & 0 & 0 & & & & & \\
\hline \multirow[t]{2}{*}{ (4) Prix de vente } & 0,42 & 0,43 & 0,28 & 1 & & & \\
\hline & 0 & 0 & 0 & & & & \\
\hline \multirow[t]{2}{*}{ (5) Nombre de visites (vulgarisation) } & 0,05 & 0,07 & 0,2 & $-0,02$ & 1 & & \\
\hline & $-0,58$ & $-0,46$ & $-0,02$ & $-0,79$ & & & \\
\hline \multirow[t]{2}{*}{ (6) Expérience } & 0,2 & 0,2 & 0,26 & 0 & 0,28 & 1 & \\
\hline & $-0,02$ & $-0,03$ & 0 & $-0,97$ & 0 & & \\
\hline \multirow[t]{2}{*}{ (7) Surface cultivée } & 0,53 & 0,54 & 0,86 & 0,23 & 0,19 & 0,33 & 1 \\
\hline & 0 & 0 & 0 & $-0,01$ & $-0,03$ & 0 & \\
\hline
\end{tabular}

Note : Les nombres qui sont entre parenthèses sont des probabilités ( $p$-values).

À côté des caractéristiques socioéconomiques des producteurs et de l'analyse corrélationnelle, les résultats détaillés d'analyse de la rentabilité de la production de la pomme de terre sont présentés dans le tableau 3. Le revenu total (RT) moyen des producteurs de pomme de terre est estimé en moyenne à 1.677.183 FRW par hectare (914.030 FRW pour les producteurs moins performants, 1.976.113 FRW pour les producteurs moyennement performants et 3.038.935 FRW pour les producteurs plus performants), alors que le coût total de production (CT) moyen est évalué à 1.025.359 FRW par hectare (à raison de 981.711 FRW pour les moins performants, 1.052.764 FRW pour les producteurs moyennement performants, et 1.058.753 FRW pour les plus performants). Le profit (ou la perte) est la différence entre le RT et le CT et est en moyenne estimé à 651.824 FRW par hectare. Les calculs montrent que 30 sur 61 producteurs dans la catégorie des moins performants réalisent un revenu net négatif $(\mathrm{RN}<0)$. Ce résultat semble dû au fait de la comptabilisation de la main d'œuvre familiale, bien que ceci ne soit pas la pratique normale des petits producteurs. Il serait aussi dû à la variabilité très hautement significative de la productivité des terres (rendement ou production par hectare), et du revenu total (ou valeur de la production, ou chiffre d'affaires). La variabilité très hautement significative de la productivité des terres serait 
expliquée par les différences des rendements (issues principalement des différences de fertilité des sols et de technicité dans la conduite des cultures), ainsi que par les différences des chiffres d'affaires (vu que les producteurs moins performants ont des rendements plus faibles que les producteurs performants).

Il est aussi remarquable que le rendement (ou productivité de la terre) chez les producteurs moins performants se situe autour de la moitié de celui des producteurs moyennement performants, et autour d'un tiers du rendement des producteurs plus performants, avec une moyenne de $8.173 \mathrm{Kg}$ par hectare. Quant à la productivité du travail (de la main d'œuvre), elle s'élève à 730 FRW chez les producteurs moins performants, à 5.349 FRW chez les producteurs moyennement performants, à 10.046 FRW chez les producteurs plus performants, et à 4.420 FRW en moyenne. Avec la variabilité très hautement significative entre les trois catégories des producteurs, les résultats montrent aussi que les producteurs de pomme de terre réalisent en moyenne une VA de 900.092 FRW par hectare (c'est-à-dire 167.968 FRW pour les producteurs moins performants, 1.188.933 FRW pour les producteurs moyennement performants et 2.193.110 FRW pour les producteurs plus performants), une MB de 692.660 FRW par hectare (à raison de -35.663 FRW pour les producteurs moins performants, 979.393 FRW pour les producteurs moyennement performants et 1.977.274 FRW pour les producteurs plus performants), un RN de 651.824 FRW par hectare (à concurrence de -67.681 FRW pour les producteurs moins performants, 925.610 FRW pour les producteurs moyennement performants et 1.957.640 FRW pour les producteurs plus performants). On a constaté également que les grandes exploitations génèrent plus de marge que les petites. 
La rentabilité de la production de pomme de terre dans la région des sols de ...

Tableau 3. Analyse de la rentabilité de la pomme de terre dans la région des sols de laves au Rwanda. 
Tropicultura Tropicultura 2295-8010 Volume 39 (2021) Numéro 4, 1909

\begin{tabular}{|c|c|c|c|c|}
\hline Indicateurs & $\begin{array}{l}\text { Pour les producteurs } \\
\text { moins performants } \\
(\mathrm{n}=61)\end{array}$ & $\begin{array}{l}\text { Pour les producteurs } \\
\text { moyennement } \\
\text { performants }(n=60)\end{array}$ & $\begin{array}{l}\text { Pour les } \\
\text { producteurs plus } \\
\text { performants }(n=11)\end{array}$ & $\begin{array}{l}\text { Pour tous les } \\
\text { producteurs } \\
(\mathrm{n}=132)\end{array}$ \\
\hline Production $(\mathrm{Kg}) * * *$ & 1.390 & 3.682 & 8.782 & 3.048 \\
\hline $\begin{array}{l}\text { Terre cultivée (ha) } \\
\text { *** }\end{array}$ & 0,28 & 0,39 & 0,62 & 0,36 \\
\hline $\begin{array}{l}\text { Rendement (Kg par } \\
\text { hectare) } * * *\end{array}$ & 5.474 & 9.715 & 14.733 & 8.173 \\
\hline $\begin{array}{l}\text { Prix de vente (FRW) } \\
\text { par } \mathrm{Kg} * * *\end{array}$ & 184 & 209 & 215 & 198 \\
\hline $\begin{array}{l}\text { Revenu total (RT) en } \\
\text { FRW *** }\end{array}$ & 255.929 & 770.684 & 1.884 .140 & 603.786 \\
\hline $\mathrm{RT} / \mathrm{ha} * * *$ & 914.030 & 1.976 .113 & 3.038 .935 & 1.677 .183 \\
\hline $\begin{array}{l}\text { Revenu total de } \\
\text { main-d'œuvre en } \\
\text { FRW }(\triangle) * * *\end{array}$ & 41.601 & 437.404 & 1.344 .395 & 330.078 \\
\hline $\begin{array}{l}\text { Main-d'œuvre } \\
\text { (nombre d'homme- } \\
\text { jours) }\end{array}$ & 57 & 82 & 134 & 75 \\
\hline $\begin{array}{l}\text { Productivité de la } \\
\text { main-d'œuvre (FRW) }\end{array}$ & 730 & 5.349 & 10.046 & 4.421 \\
\hline $\begin{array}{l}\text { Coût total variable } \\
\text { (CTV) en FRW }\end{array}$ & 265.914 & 388.721 & 658.230 & 354.428 \\
\hline CTV/ha & 1.030 .553 & 1.003 .545 & 1.037 .762 & 984.523 \\
\hline $\begin{array}{l}\text { Consommations } \\
\text { intermédiaires (CI) } \\
\text { en FRW *** }\end{array}$ & 208.898 & 306.934 & 524.411 & 279.753 \\
\hline CI/ha en FRW ${ }^{\text {ns }}$ & 789.154 & 787.893 & 819.655 & 791.123 \\
\hline $\begin{array}{l}\text { Plants de pomme de } \\
\text { terre (FRW) }\end{array}$ & 99.339 & 141.195 & 242.636 & 130.306 \\
\hline $\begin{array}{l}\text { Engrais organiques } \\
\text { (FRW) }\end{array}$ & 21.533 & 29.383 & 25.364 & 25.421 \\
\hline $\begin{array}{l}\text { Engrais chimiques } \\
\text { (FRW) }\end{array}$ & 62.041 & 93.293 & 93.293 & 93.293 \\
\hline
\end{tabular}


La rentabilité de la production de pomme de terre dans la région des sols de ...

\begin{tabular}{|c|c|c|c|c|}
\hline Pesticides (FRW) & 15.487 & 20.230 & 36.236 & 19.372 \\
\hline Transport (FRW) & 10.498 & 22.833 & 22.833 & 18.016 \\
\hline $\begin{array}{l}\text { Valeur ajoutée en } \\
\text { FRW (VA) *** }\end{array}$ & 47.031 & 463.684 & 1.359 .728 & 324.033 \\
\hline VA/ha en FRW $* * *$ & 167.968 & 1.188 .933 & 2.193 .110 & 900.092 \\
\hline Main d'œuvre (FRW) & 57.016 & 81.767 & 133.818 & 74.667 \\
\hline $\begin{array}{l}\text { Marge brute (MB) en } \\
\text { FRW *** }\end{array}$ & -9.986 & 381.963 & 1.225 .910 & 249.358 \\
\hline $\mathrm{MB} / \mathrm{ha}$ en FRW $* * *$ & -35.663 & 979.393 & 1.977.274 & 692.660 \\
\hline $\begin{array}{l}\text { Coût total fixe (CTF) } \\
\text { en FRW ** }\end{array}$ & 8.965 & 20.975 & 12.285 & 14.701 \\
\hline CTF/ha en FRW ${ }^{\text {ns }}$ & 44.371 & 49.219 & 20.992 & 44.626 \\
\hline Rente (FRW) & 2.377 & 13.500 & 2.955 & 7.481 \\
\hline Dépréciation (FRW) & 6.588 & 7.475 & 9.330 & 7.220 \\
\hline $\begin{array}{l}\text { Coût total (CT) en } \\
\text { FRW *** }\end{array}$ & 274.879 & 409.696 & 670.514 & 369.129 \\
\hline CT/ha en FRW ${ }^{\text {ns }}$ & 981.711 & 1.052 .764 & 1.058 .753 & 1.025 .359 \\
\hline $\begin{array}{l}\text { Revenu net }(\mathrm{RN}) \text { en } \\
\text { FRW *** }\end{array}$ & -18.951 & 360.988 & 1.213 .625 & 234.657 \\
\hline $\mathrm{RN} / \mathrm{ha}$ en FRW $* * *$ & -67.681 & 925.610 & 1.957 .460 & 651.824 \\
\hline
\end{tabular}

*** $\mathrm{p}<0,01$ (différence très hautement significative), ** $\mathrm{p}<0,05$ (différence hautement significative), * $\mathrm{p}<0,1$ (différence significative) entre les trois catégories de producteurs ; "ns" signifie différence non significative ; ha=hectare ; (๑) Revenu total du travail (main-d'œuvre)=RT-(CT-Dépenses sur la main-d'œuvre).

Note : taux de change d'un dollar US en francs rwandais est de 899,45 FRW (taux de change annuel moyen calculé à l'aide des publications de la Banque Nationale du Rwanda pour l'année 2019).

En plus de l'analyse des composantes du coût de production et des indicateurs de productivité et de rentabilité (tableau 3), nous avons examiné la sensibilité de la rentabilité des exploitations agricoles à une baisse de la production agricole ou à une hausse des coûts de production. En adoptant les principes de l'analyse marginaliste, nous avons considéré des taux de variation de $1 \%$ (47). Les résultats de l'analyse (tableau 4) montrent que la perte de la production de $1 \%$ résulterait en une diminution de $1 \%$ du revenu total par hectare (ce qui revient à une diminution de 1,0 \% pour les producteurs moins performants, de 1,0 \% pour les producteurs moyennement performants et de 1,0 \% pour les producteurs plus performants) et une baisse de 1,9\% de la valeur ajoutée par hectare 
(ce qui revient à une diminution de 1,0\% pour les producteurs moins performants, de 1,0\% pour les producteurs moyennement performants et de 1,0\% pour les producteurs plus performants). Il en résulterait également un déclin de 2,42\% de la marge brute par hectare (soit un déclin de 25,6\% pour les producteurs moins performants, de 2,1\% pour les producteurs moyennement performants et de 1,5\% pour les producteurs plus performants), et un abaissement de 2,6 \% du revenu net par hectare (soit une baisse de 13,5\% pour les producteurs moins performants, de 2,2 \% pour les producteurs moyennement performants et de 1,6 \% pour les producteurs plus performants). 
La rentabilité de la production de pomme de terre dans la région des sols de ...

Tableau 4. Analyse de sensibilité de la rentabilité de la pomme de terre au choc de la production. 
Tropicultura Tropicultura 2295-8010 Volume 39 (2021) Numéro 4, 1909

\begin{tabular}{|c|c|c|c|c|}
\hline Indicateurs & $\begin{array}{l}\text { Les producteurs } \\
\text { moins performants } \\
(n=61)\end{array}$ & $\begin{array}{l}\text { Les producteurs } \\
\text { moyennement } \\
\text { performants }(n=60)\end{array}$ & $\begin{array}{l}\text { Les producteurs } \\
\text { plus performants } \\
(\mathrm{n}=11)\end{array}$ & $\begin{array}{l}\text { Ensemble des } \\
\text { producteurs } \\
(\mathrm{n}=132)\end{array}$ \\
\hline Production (Kg) & 1.376 & 3.645 & 8.694 & 3.017 \\
\hline $\begin{array}{l}\text { Revenu total (RT) en } \\
\text { FRW }\end{array}$ & 253.370 & 762.645 & 1.865 .288 & 597.747 \\
\hline Terre cultivée (ha) & 0,28 & 0,39 & 0,62 & 0,36 \\
\hline $\begin{array}{l}\text { RT par hectare } \\
\text { (FRW) }\end{array}$ & 904.891 & 1.955 .645 & 3.008 .529 & 1.660 .409 \\
\hline $\begin{array}{l}\text { Consommations } \\
\text { intermédiaires (CI) } \\
\text { en FRW }\end{array}$ & 208.898 & 306.934 & 524.411 & 279.753 \\
\hline $\begin{array}{l}\text { Coût total variable } \\
\text { (CTV) en FRW }\end{array}$ & 265.914 & 388.721 & 658.230 & 354.428 \\
\hline $\begin{array}{l}\text { Valeur ajoutée (VA) } \\
\text { en FRW }\end{array}$ & 44.472 & 455.710 & 1.340 .876 & 317.995 \\
\hline $\begin{array}{l}\text { VA par hectare en } \\
\text { FRW }\end{array}$ & 158.527 & 1.168 .488 & 2.162 .704 & 883.319 \\
\hline $\begin{array}{l}\text { Marge brute (MB) en } \\
\text { FRW }\end{array}$ & -12.545 & 373.924 & 1.207 .058 & 243.319 \\
\hline $\begin{array}{l}\text { MB par hectare en } \\
\text { FRW }\end{array}$ & -44.803 & 958.779 & 1.946 .868 & 675.886 \\
\hline $\begin{array}{l}\text { Coût total (CT) en } \\
\text { FRW }\end{array}$ & 274.879 & $409.696,20$ & 670.514 & 369.129 \\
\hline $\begin{array}{l}\text { Revenu net }(\mathrm{RN}) \text { en } \\
\text { FRW }\end{array}$ & -21.510 & 352.948 & 1.194 .773 & 228.618 \\
\hline $\begin{array}{l}\text { RN par hectare } \\
\text { (FRW) }\end{array}$ & -76.820 & 904.996 & 1.927 .054 & 635.051 \\
\hline Variations & & & & \\
\hline Variation RT /ha * & -9.139 & -20.468 & -30.406 & -16.773 \\
\hline Variation RT/ha ( \%) & -1 & $-1,04$ & $-1,01$ & -1 \\
\hline Variation VA/ha ( \%) & $-5,44$ & $-1,72$ & $-1,39$ & $-1,86$ \\
\hline Variation MB/ha ( \%) & $-25,63$ & $-2,1$ & $-1,54$ & $-2,42$ \\
\hline
\end{tabular}


La rentabilité de la production de pomme de terre dans la région des sols de ...

Variation RN/ha ( \%) $-13,5$

$-2,23$

$-1,55$

Note : * En valeur absolue, le RT/ha, la VA/ha, la MB/ha, et le RN/ha varient d'un même montant dans l'ensemble et au niveau de chaque groupe de producteurs.

En procédant toujours par l'analyse marginaliste, nous avons examiné les effets de l'augmentation des coûts de production sur la rentabilité. Les coûts totaux par hectare étant du même ordre de grandeur entre les 3 groupes (voir le tableau 3), les variations sur les différents indicateurs prises en valeur absolue sont aussi du même ordre entre les 3 groupes du fait que leur variabilité n'est pas significative. Par contre, la structure des coûts n'est pas identique. Nous avons de ce fait trouvé intéressant d'analyser les effets d'une hausse des coûts des engrais (organiques et chimiques) sur la rentabilité de la pomme de terre. Les résultats (tableau 5) montrent que, suite à l'augmentation du coût des engrais de $1 \%$, la VA par hectare va diminuer de 0,3 \% (ce qui correspond au niveau de 1,8\% pour les producteurs moins performants, de $0,3 \%$ pour les producteurs moyennement performants et de $0,3 \%$ pour les producteurs plus performants). Ceci résulterait à une baisse de la MB par hectare de 0,5\% (au taux de 8,4 \% pour les producteurs moins performants, de 3,3 \% pour les producteurs moyennement performants et de $0,1 \%$ pour les producteurs plus performants). Quant au RN par hectare, il y aura une diminution de 0,5\% (ce qui s'élève à une baisse de 4,4 \% pour les producteurs moins performants, $3,3 \%$ pour les producteurs moyennement performants et de $0,1 \%$ pour les producteurs plus performants). 
Tropicultura Tropicultura 2295-8010 Volume 39 (2021) Numéro 4, 1909

Tableau 5. Analyse de sensibilité de la rentabilité de la pomme de terre à l'augmentation du coût. 
La rentabilité de la production de pomme de terre dans la région des sols de ...

\begin{tabular}{|c|c|c|c|c|}
\hline Indicateurs & $\begin{array}{l}\text { Les producteurs moins } \\
\text { performants }(n=61)\end{array}$ & $\begin{array}{l}\text { Les producteurs } \\
\text { moyennement performants } \\
(\mathrm{n}=60)\end{array}$ & $\begin{array}{l}\text { Les producteurs plus } \\
\text { performants }(n=11)\end{array}$ & $\begin{array}{l}\text { Pour tous les } \\
\text { producteurs } \\
(n=132)\end{array}$ \\
\hline $\begin{array}{l}\text { Production } \\
(\mathrm{Kg})\end{array}$ & 1.390 & 3.682 & 8.782 & 3.048 \\
\hline $\begin{array}{l}\text { Revenu total } \\
\text { (RT) en FRW }\end{array}$ & 255.929 & 770.684 & 1.884 .140 & 603.786 \\
\hline $\begin{array}{l}\text { Coûts des } \\
\text { engrais }\end{array}$ & 84.410 & 123.903 & 119.844 & 119.901 \\
\hline $\begin{array}{l}\text { Coût total } \\
\text { (FRW) }\end{array}$ & 275.715 & 410.903 & 567.652 & 376.963 \\
\hline RT /ha (FRW) & 914.030 & 1.976 .113 & 3.038 .935 & 1.677 .183 \\
\hline CI /ha (FRW) & 749.049 & 790.156 & 679.917 & 798.875 \\
\hline $\begin{array}{l}\text { CTV /ha } \\
(\mathrm{FRW})\end{array}$ & 959.191 & 1.006 .687 & 1.072 .277 & 994.368 \\
\hline $\begin{array}{l}\text { VA par } \\
\text { hectare } \\
(\text { FRW })\end{array}$ & 160.506 & 1.181 .232 & 2.184 .652 & 892.321 \\
\hline $\begin{array}{l}\text { MB par } \\
\text { hectare } \\
\text { (FRW) }\end{array}$ & -45.160 & 969.426 & 1.966 .658 & 682.815 \\
\hline CT /ha (FRW) & 984.696 & 1.053 .597 & 915.567 & 1.047 .120 \\
\hline $\begin{array}{l}\text { RN par } \\
\text { hectare } \\
(\text { FRW })\end{array}$ & -70.666 & 922.464 & 1.955 .546 & 648.526 \\
\hline Variations & & & & \\
\hline $\begin{array}{l}\text { Variation VA } \\
\text { /ha } \mathrm{R}\end{array}$ & -2.985 & -3.146 & -1.914 & -3.298 \\
\hline $\begin{array}{l}\text { Variation VA/ } \\
\text { ha ( } \%)\end{array}$ & $-1,78$ & $-0,25$ & $-0,13$ & $-0,34$ \\
\hline $\begin{array}{l}\text { Variation } \\
\text { MB/ha ( \%) }\end{array}$ & $-8,37$ & $-3,32$ & $-0,09$ & $-0,49$ \\
\hline $\begin{array}{l}\text { Variation RN/ } \\
\text { ha ( \%) }\end{array}$ & $-4,41$ & $-3,34$ & $-0,07$ & $-0,51$ \\
\hline
\end{tabular}


Note : Les coûts totaux par hectare étant du même ordre de grandeur entre les 3 groupes, nous avons jugé utile d'analyser les effets d'une hausse des coûts des engrais (organiques et chimiques) sur la rentabilité des petits producteurs. R Les montants en valeur absolue du RT/ha, de la VA/ha, de la MB/ha, et du RN/ha varient d'un même montant dans l'ensemble et au niveau de chaque groupe de producteurs.

L'analyse de rentabilité de la production de la pomme de terre et de sa sensibilité à la diminution de la production ainsi qu'à l'augmentation du coût de production a été complétée par la présentation de la structure des coûts de production. La figure 3 montre la variation des composantes des coûts entre les producteurs moins performants (figure 3.a), les producteurs moyennement performants (figure 3.b), et les producteurs plus performants (figure 3.c). Ces résultats peuvent nous conduire à penser que les deux premières catégories de producteurs de pomme de terre (figure 3. a et b) exploitent les terres marginales, du fait qu'ils affectent $30 \%$ de leurs coûts de production à l'acquisition des engrais (chimiques et organiques) contre $20 \%$ pour la troisième catégorie (figure 3. c). En plus de cela, il semble que les producteurs les plus performants préfèrent les plants de pomme de terre de meilleure qualité : ils y affectent $43 \%$ de leur coût de production, contre $36 \%$ et $34 \%$, respectivement pour les producteurs moins performants et les producteurs moyennement performants.

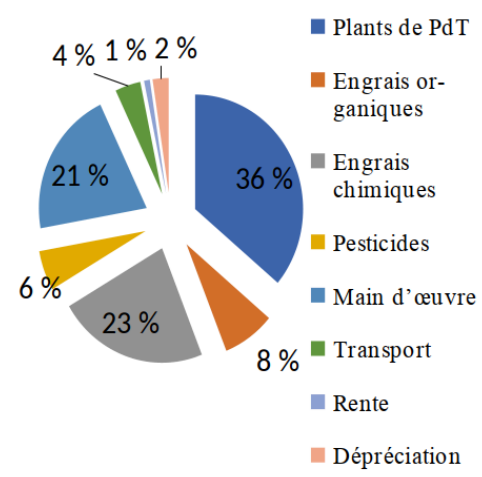

a Producteurs moins performants

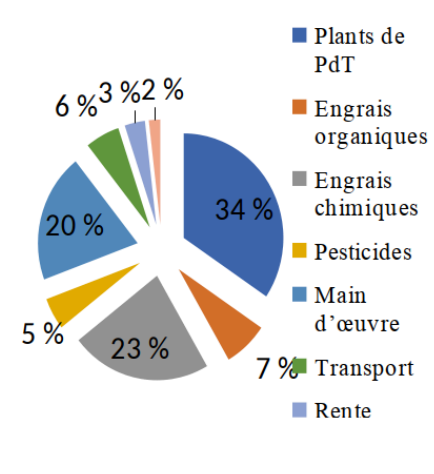

b. Producteurs moyennement performants

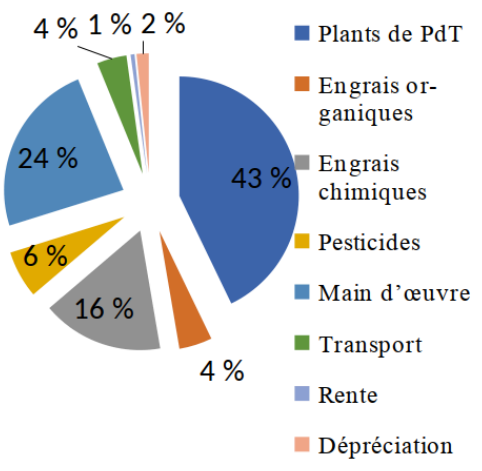

c Producteurs plus performants

Note : PdT=pomme de terre

\section{Figure 3. Structure des coûts de production des trois catégories des producteurs de} pomme de terre

\section{Discussion}

La pomme de terre procure des revenus aux agriculteurs bien qu'elle ne soit pas profitable à tous. Sa rentabilité est mesurée par le revenu net positif ou négatif obtenu par la différence entre le revenu total (valeur de la production) et le coût de production total (25). Pour les producteurs non performants, leur niveau de rentabilité pourrait être contraint par le coût élevé de la production (23) dont la source serait l'inefficacité dans l'usage des ressources disponibles pour les exploitants agricoles ou potentiellement par l'augmentation du prix des intrants. Cela serait aussi expliqué par la variabilité, entre les trois catégories de producteurs, du niveau des productivités de la terre et du 
La rentabilité de la production de pomme de terre dans la région des sols de ...

travail qui s'élèvent en moyenne à $8.173 \mathrm{~kg}$ par hectare (au niveau de $5.474 \mathrm{~kg}$ pour les producteurs moins performants, $9.715 \mathrm{~kg}$ pour les producteurs moyennement performants et $14.733 \mathrm{~kg}$ pour les producteurs plus performants) et à $4.420 \mathrm{FRW}$ (estimé à $730 \mathrm{FRW}$ pour les producteurs moins performants, 5.349 FRW pour les producteurs moyennement performants et 10.046 FRW pour les producteurs plus performants), respectivement.

Comme les résultats de l'analyse corrélationnelle ont mis en évidence l'effet significatif du coût de production, de l'expérience du producteur, et de la surface des terres exploitées sur le niveau de rentabilité de la pomme de terre (mesurée par le revenu net), il s'avère nécessaire pour les exploitants de choisir les techniques agricoles nouvelles et innovantes qui augmentent la productivité et l'efficacité de la production $(17,21)$. Au vu de ce que l'analyse de sensibilité suggère, l'effet d'une perte de production de $1 \%$ impacte davantage la valeur ajoutée par hectare $(-1,86 \%)$ et la marge brute par hectare $(-2,42 \%)$ qu'une augmentation des coûts de $1 \%(-0,86 \%$ et $-1,42 \%$, respectivement pour la valeur ajoutée par hectare et la marge brute par hectare). Ces différences sont implicites vu qu'une diminution de $1 \%$ du rendement a un effet plus marqué qu'une hausse de $1 \%$ des coûts de production (via l'augmentation des coûts des engrais organiques et chimiques). La rentabilité de la pomme de terre s'est aussi montrée sensible à la diminution inattendue de la production qui pourrait être dûe aux différents risques agricoles (31). Les exploitants doivent bien connaître ces risques et par-là contrôler la sensibilité de la production à un certain nombre de facteurs susceptibles de causer une diminution de la rentabilité (23). Spécifiquement pour le coût de production, la relation positive avec le revenu agricole net justifie la nécessité des investissements dans les activités de production agricole (14). De plus, étant donné que la rentabilité est aussi plus sensible au niveau du coût de production, la maîtrise de celui-ci serait aussi un déterminant majeur de la rentabilité de la pomme de terre $(2,6,36)$. Ceci demanderait à certains producteurs de prêter attention à leurs spécificités (fertilité du sol, qualité des plants de pomme de terre à choisir, par exemple) lors des décisions sur les investissements et les affectations de fonds.

Les résultats de notre recherche montrent que la production de la pomme de terre dans la région des sols de laves au Rwanda est toujours rentable, sauf pour $23 \%$ de mon échantillon. En prix constants de 2009 (prix de vente estimé à 96 FRW par kilo de pomme de terre, et le coût unitaire de production de $68 \mathrm{FRW}$ ), les coûts variables sont respectivement 700.313 FRW en 2009 et 556.254 FRW en 2019 (par notre étude), alors que les revenus totaux seraient respectivement de 991.829 FRW et de 784.608 FRW. Dans ces conditions, les marges brutes seraient de 291.516 FRW en 2009 et 228.354 FRW en 2019 et, par conséquent, la production de la pomme de terre serait toujours rentable, mais apparemment moins qu'en 2009. Si elle remplit toutes ces conditions, la pomme de terre maintiendra sa qualification de culture de sécurité alimentaire (37).

\section{Conclusions}

La pomme de terre joue un rôle très prépondérant dans la nutrition humaine qui est fortement préconisée par la FAO. Grâce à son haut rendement et sa courte saison de croissance, cette culture permet aux petits exploitants de répondre aux obstacles de faim et de malnutrition. Pour ce faire, la production agricole doit assurer des revenus en croissance stable aux ménages de producteurs, ce qui découle aussi de l'adoption et de l'usage approprié de technologies favorisant la croissance de la productivité, dont le résultat serait l'augmentation des revenus, la disponibilité des produits agricoles moins chers pour les pauvres, ainsi que le développement d'activités économiques non agricoles surtout dans les milieux ruraux. Face à ces attentes, la pomme de terre est considérée comme une culture capable de contribuer à l'amélioration des conditions de vie des exploitants, 
mais le problème se situe essentiellement au niveau de la variabilité du prix de vente et du coût de production élevé.

Les résultats de l'analyse budgétaire mettent en évidence que la production de la pomme de terre est rentable sous certaines conditions et que la rentabilité est sensible à la diminution de l'offre (production) et à l'augmentation du coût des engrais organiques et chimiques. Pour améliorer le niveau de rentabilité, les producteurs de pomme de terre doivent pratiquer les méthodes agricoles qui renforcent la productivité. L’analyse par la méthode corrélationnelle a mis en évidence que le coefficient est positif et significatif entre le revenu agricole net et les autres facteurs, notamment le coût de production, le prix de vente, l'expérience de l'exploitant et la surface des terres exploitées. Ces variables sont des facteurs qu'il faut considérer pour maintenir et/ou améliorer le niveau de rentabilité agricole. Par comparaison, l'analyse de la sensibilité du revenu agricole net montre que les effets du choc de la production sont plus néfastes que ceux de l'augmentation du coût de production.

Vu que la superficie des terres est très petite, le Gouvernement du Rwanda doit poursuivre la limitation du morcellement des terres et le changement d'usage des terres cultivables afin d'éviter de se situer dans une tendance structurelle lourde qui pourrait affecter les revenus des producteurs de la pomme de terre. Dans ce cadre, la planification des usages des terres doit être repensée et/ou réadaptée aux dynamiques démographique et économique pour protéger la terre contre les enjeux climatiques. Il se pose également le souci de la durabilité du système de production des pommes de terre, de conservation de la fertilité des sols, ce qui fait appel à l'adoption des variétés des plants de pomme de terre adaptées à haut rendement, à une utilisation raisonnée des fertilisants et des pesticides en valorisant autant que possible la fumure organique et la lutte biologique, etc. Ces points seraient importants pour comprendre la durabilité environnementale du système de production de la pomme de terre dans la région agricole des sols de laves au Rwanda. La littérature existante et les recherches ultérieures pourraient expliquer cette dimension du développement.

Sur base de toutes ces discussions, nous recommandons aux producteurs de pomme de terre de s'efforcer d'adopter les pratiques agricoles qui réduisent les inefficacités technico-économiques. Quant aux autorités publiques, elles devraient veiller à ce que les exploitants agricoles puissent s'approvisionner en variétés de plants de pomme de terre à haut rendement adaptées aux conditions agro-écologiques ainsi que des services de vulgarisation répondant efficacement aux besoins réels des producteurs.

\section{Bibliographie}

1. AFSR, 2007, Système de gestion de la Filière Semencière du Rwanda - > Module 4 : Savoir-faire technique : Fiche technique de production > des semences certifiées : pomme de terre. Kigali, AFSR (Appui à > la Filière Semencière au Rwanda), 5 pages.

2. Alinda F. \& Abbott P., 2012, Agricultural policy and institutional > framework for transformation of agriculture, economic development $>$ and poverty reduction in Rwanda. Institute of Policy Analysis and > Research-Rwanda, Kigali, 38 pages.

3. Barker A., 2000, La pomme de terre. Plus de 150 recettes de plats $>$ et de salades. Manisse (les Editions Minerva), Genève, 258 pages.

4. Bouquiaux J. M., Vanorlé L., Daniel R., Buron M. H., \& Delille N. > (2009). Performances 
La rentabilité de la production de pomme de terre dans la région des sols de ...

et rentabilité en agriculture wallonne. Les > livrets de l’ agriculture, (18).

5. Bublot G., 1965, Exploitation agricole. Économie-Gestion-Analyse. > Éditions Nauwelaerts, Louvain, Belgique.

6. Cervantes-Godoy D. \& Dewbre J., 2010, Importance économique de > l'agriculture dans la lutte contre la pauvreté. Food, Agriculture > and Fisheries Working Papers No. 23. Editions OECD, Paris, 29 > pages.

7. CIDSE, 2012, Agriculture : du Problème à la Solution - Garantir le > Droit à l'Alimentation dans un Monde de Contraintes Climatiques. > Coopération Internationale pour le Développement et la Solidarité > (CIDSE), Bruxelles, 24 pages.

8. CIRAD, 2013, Les agricultures familiales du monde : Définitions, > contributions et politiques publiques. Centre de Coopération > Internationale en Recherche Agronomique pour le Développement > (CIRAD), Montpellier, 306 pages.

9. Cochet H., 2015, Comparative agriculture. Springer, London, $154>$ pages.

10. Corselius K., Wisniewski S. \& Ritchie M., 2001, Sustainable > agriculture: making money, making sense.The Institute for > Agriculture and Trade Policy, Washington DC, 48 pages.

11. Devaux A., Kromann P. \& Ortiz O., 2014, Potatoes for sustainable > global food security. Potato Research, 57(3-4), 185-199.

12. Dorward A. \& Kydd J., 2005, Fonctionnement des marchés agricoles en > faveur des plus pauvres. L'actualité des services aux entreprises > No. 9. Un produit d'information de la DGCID (MAE), 8 pages.

13. FAO, 2009, International year of the potato 2008: light on a hidden > treasure. Rome, United Nations Food and Agriculture Organization, > 148 pages.

14. FAO, 2012, La situation mondiale de l'alimentation et de > l'agriculture : investir dans l'agriculture pour un avenir > meilleur. Organisation des Nations Unies pour l'Alimentation et > l'Agriculture (FAO), Rome, 202 pages.

15. Frans G., 2002, Potato Marketing in Rwanda. Agricultural Policy > Development Project Research Report, (12), 38-56.

16. Gietema B. (Ed.), 2006, Farm accounting. Agromisa Foundation, > Wageningen, 50 pages.

17. Gnanglè P. C., Afouda Yabi J., Yegbemey N. R., Glèlè Kakaï L. R. \& > Sokpon N., 2012, Rentabilité économique des systèmes de production > des parcs à karité dans le contexte de l'adaptation au changement $>$ climatique du Nord-Bénin. African Crop Science Journal, > 20(Supplement s2), 589 - 602.

18. GoR, 2007, Vision 2020 Umurenge. An integrated local development $>$ program to accelerate poverty eradication, rural growth, and > social protection. The Government of Rwanda (GoR), Kigali, $51>$ pages.

19. IFAD, 2013, Smallholders, food security and the environment $>$ (Report). International 
Fund for Agricultural Development (IFAD), > Rome, 54 pages.

20. IMF, 2011, Rwanda: Poverty Reduction Strategy Paper-Progress > Report. IMF Country Report No. 11/154. International Monetary > Fund (IMF), Washington DC, 266 pages.

21. Lee D. R., 2005, Agricultural sustainability and technology > adoption: Issues and policies for developing countries. American > Journal of Agricultural Economics, 87(5), 1325-1334.

22. Magain P., non daté, Introduction aux méthodes quantitatives et $>$ éléments de statistique. Cours de $3^{\mathrm{e}}$ bachelier en information $>$ et communication, Université de Liège, Belgique.

23. Maniriho A. \& Bizoza A., 2013, Financial Benefit-Cost Analysis of > Agricultural Production in Musanze District (Rwanda). Academia > Arena, 5(12), 30-39.

24. Maniriho A., 2013, The determinants of agricultural production and $>$ profitability in Musanze District, Rwanda. MSc thesis, National > University of Rwanda, Kigali, Rwanda, 82 pages. Disponible sur > http://hdl.handle.net/2268/262314

25. Mankiw G. N. \& Taylor M. P., 2010, Principe de l'économie. > Éditions De Boeck Université, Bruxelles, 1175 pages.

26. Milz M., 2010, La révolution verte au Rwanda : un programme > d'intensification autoritaire au service de l'agrobusiness. $7>$ pages. Disponible sur $>$ https://www.grain.org/media/ BAhbBlsHOgZmSSI+MjAxMS8wOC8wOC8xMF8wNF80MF82MTFfTGFfcl92b2x1dGlvbl92ZXJ0ZV9hdV9S La > \%20r \%C3 \%A9volution \%20verte \%20au > \%20Rwanda.pdf > (consulté le 26/10/ 2019).

27. MINAGRI, 2018, Strategic plan for agriculture transformation > 2018-2024. Ministry of Agriculture and Animal Resources > (MINAGRI), Kigali, Rwanda, 235 pages.

28. MINECOFIN, 2012, Economic Development and Poverty Reduction > Strategy 2013-2018. Ministry of Finance and Economic Planning, > Kigali, Rwanda, 172 pages.

29. Mpyisi E., 2002, Estimation of Area and Production of Root and > Tuber Crops in Rwanda. Paper presented at the FAO Expert > Consultation on Root Crop Statistics, held in Harare, Zimbabwe, > December 2-6, 2002.

30. Mugabe J., Tuyisenge J., Badege P., Mutware J. S., Mugabo J. R. \& > Nyirigira A. M., 2009, Estimating the profitability of major food > crops in Rwanda. Institut des Sciences Agronomiques au Rwanda > (ISAR), Kigali, 63 pages.

31. Mulumeoderhwa-Munyakazi F., Maniriho A., Manirakiza D., Mastaki J. > L. \& Lebailly P., 2019, Risk analysis in the peasant framework: > empirical analysis of farmers in South Kivu, Democratic Republic > of Congo. Agriculture \& Forestry 65(4), 35-45.

32. Musabanganji E., Maniriho A., Kayisire P. \& Nyalihama C., 2019, > Regional Trade and Competitiveness of Rwandan Agriculture: > Empirical Analysis of Selected Priority 
La rentabilité de la production de pomme de terre dans la région des sols de ...

Foodstuffs, 26 pages. > Disponible sur > http://hdl.handle.net/2268/238384

33. Ndindabahizi I. \& Ngwabije R., 1991, Stratégie nationale de > conservation des sols: Evaluation des systèmes d'exploitation > agricole pour une régionalisation des techniques de conservation > et d'aménagement des fertilités des sols. Rapport d'une mission > de consultation réalisée du 15-01-1991. Kigali: Ministère de > l’Agriculture, de l'élevage et des forêts, 147 pages.

34. NISR, 2014, Fourth Population and Housing Census, Rwanda, 2012 - > Final results Main indicators report. National Institute of $>$ Statistics of Rwanda (NISR), Kigali, 171 pages.

35. NISR, 2018, Season Agricultural Survey 2018 Annual Report. > National Institute of Statistics of Rwanda (NISR), Kigali, 95 > pages.

36. Nuwagira S., 2017, New project: How Rwanda plans to be a global > potato exporter. The New Times Rwanda (August 22, 2017). > Retrieved from > https://www.newtimes.co.rw/ section/read/218441

37. Okello J. J., Zhou Y., Kwikiriza N., Ogutu S., Barker I., > Schulte-Geldermann E., ... \& Ahmed J. T., 2017, Productivity and $>$ food security effects of using of certified seed potato: the case > of Kenya's potato farmers. Agriculture \& Food Security 6(1), > 25-33.

38. RCA, 2009, Umurenge SACCO strategy. Kigali, Rwanda Cooperative > Agency (RCA), 51 pages.

39. RIU Rwanda, 2010, Potato innovation platform. Source: http:// > www.researchintouse.com/programmes/riu-rwanda/riu-rw42innovplatpotato.html > consulté le 10 avril 2018.

40. Rushemuka P. N., Bock, L. \& Mowo J. G., 2014, Soil science and > agricultural development in Rwanda: state of the art. A review. > Biotechnology, Agronomy, Society and Environment, 18(1), > 142-154.

41. Rwanda Environment Management Authority (REMA), 2009, Rwanda State > of Environment and Outlook report. Rwanda Environment Management > Authority, Kigali, Rwanda, 177 pages.

42. Sanginga N. \& Mbabu A., 2015, Root and tuber crops (cassava, yam, > potato and sweet potato). Abidjan, African Development Bank, 29 > pages.

43. Scott G. J., 1988, Potatoes in Central Africa: A Study of Burundi, > Rwanda, and Zaire. Lima, International Potato Centre, 159 pages.

44. Shaaban H. \& Kisetu E., 2014, Response of Irish Potato to NPK > fertilizer application and its economic return when grown on an > Ultisol of Morogoro, Tanzania. Journal of Agriculture and Crop > Resources 2(9), 188-196.

45. Sourisseau J. M., Kahane R., Fabre P. \& Hubert B. (Eds.), 2015, > Actes des Rencontres internationales: Agricultures familiales et > recherche (Ateliers, Montpellier, 1-3 Juin 2014). Montpellier, > Agropolis International, 320 pages. 
46. Walras L., 1926, Eléments d'économie politique pure ou théorie de > la richesse sociale (Édition définitive, revue et augmentée par > l'auteur). R. Pichon et R. Durand-Auzias (Editeurs), Paris, $499>$ pages.

47. Ward J. H., 1963, Hierarchical grouping to optimize an objective $>$ function. Journal of the American Statistical Association, > 58(301), 236-244.

\section{Notes}

$1 \mathrm{Au}$ Rwanda, une année agricole comprend trois saisons culturales, notamment la saison A qui va de septembre à février, la saison B de mars à juin, et la saison C de juillet à septembre.

$\underline{2}$ Le Rwanda est organisé administrativement par ordre hiérarchique en 5 provinces (y compris la Ville de Kigali), 30 districts, 416 secteurs, 2.148 cellules, et 14.837 villages. Une cellule est une unité administrative en-dessous du secteur et au-dessus du village.

PDF généré automatiquement le 2023-04-26 06:45:12

Url de l'article : https://popups.uliege.be/2295-8010/index.php?id=1909 